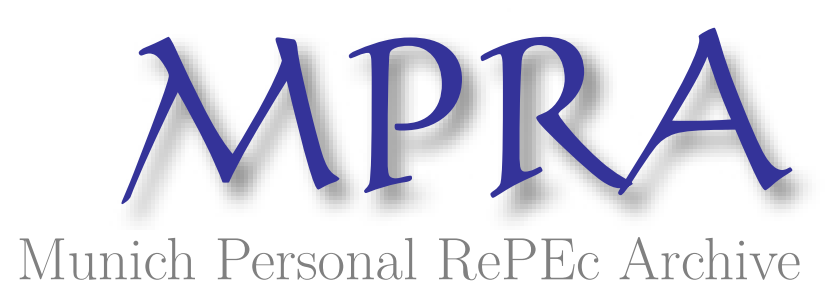

\title{
Adverse selection and optimum insurance policies
}

Sheshinski, Eytan

The Hebrew University of Jerusalem

1977

Online at https://mpra.ub.uni-muenchen.de/70329/

MPRA Paper No. 70329, posted 13 Jun 2016 14:38 UTC 


\title{
Adverse selection and optimum insurance policies
}

\author{
Eytan Sheshinski*
}

June 9, 2016

\begin{abstract}
This paper considers how insurance companies choose a price schedule for policies depending on the size of these policies which are determined by households. Under asymmetric information, we analyse the tension between self-selection and the density distribution of household by accident probability. The profit maximizing policy is compared to the socially optimal policy.
\end{abstract}

JEL Classification: H21.H23, H31, H43, H42

Key Word: Adverse Selection, Insurance, Pareto efficiency, Optimal pricing

*Department of Economics, The Hebrew University of Jerusalem. e-mail: msey$\tan @$ mscc.huji.ac.il 


\section{Introduction}

We describe here how an insurance company decides on the price schedule for insurance contracts against certain accidents (or medical needs) which it offers on the basis of the choices made by the customers themselves regarding the contracts that they purchase. The return from an insurance contract is a random variable which depends on the accident probability of the individual customer. While an individual may generally be assumed to be well-informed about his accident probability, the insurance firm has only imperfect information about this probability and, consequently, can only observe the relation between the type of insurance bought by different customers and their accident frequencies. This paper is concerned with the effect of such self-selecting information devices on the policy chosen by a profit maximizing firm. ${ }^{1}$

We consider an insurance firm that establishes a price schedule for insurance contracts and allows customers indiscriminantly to determine the size of the contract. Accident-prone individuals are expected to purchase relatively large insurance policies, and may typically be willing to pay a higher price for them. It has thus been argued that this "adverse selection" aspect will induce firms to charge proportionately higher prices for large contracts. This argument disregards, however, the effect of the distribution of customers (by accident probabilities) on the optimum policy. If a sufficiently large number of customers is concentrated in the high-probabilities range, it may be profitable, depending on demand characteristics, not to charge too high a price for large contracts. We first consider a simple case in which the firm chooses a profit-maximizing linear policy, setting a quantity constraint on the size of permitted policies. The policy chosen by the monopoly is then compared with a policy of the same type that maximizes social welfare, defined as the sum of individuals' expected utilities. It is shown that the monopoly chooses to provide a lower return on contracts, or sets a lower quantity constraint, or both, compared with the social optimum. A second example provides an explicit solution for the profit-maximizing nonlinear schedule chosen by the firm. This example suggests that the schedule need not be convex throughout, due to the interaction of demands and the distribution of customers. The performance of markets when buyers or sellers have imperfect information concerning some relevant attributes has been of much recent interest. The implications for general equilibrium of contingent insurance contracts are discussed by Rothschild and Stiglitz (1976). Other cases involving labor markets are discussed by Spence (1974), Arrow (1973), and Salop and Salop (1976). This paper extends these works by allowing for nonlinear price schedules (including quantity constraints). The results, however, pertain only to special cases and can therefore be regarded only as illustrative. In solving the nonlinear problem, we make use of the methods developed to solve a similar problem in the theory of optimum income taxation [see Mirrlees (1971) and

\footnotetext{
${ }^{1}$ Insurance firms also look, of course, for objective characteristics (health, age, etc.) which are correlated with accident probabilities, but these (costly) screening devices generally still leave a great deal of variability within each group. For simplicity, we disregard the problem of optimum investment in such examinations of customers' status.
} 
Sheshinski (1976)].

\section{The general problem}

Consider a group of individuals, all of whom have some risk of suffering an accident, which, if it occurs, does the same damage to each person. In the absence of insurance, all individuals are assumed to have the same income in each state: $y_{0}\left(y_{0}>0\right)$ if no accident occurs and $y_{1}\left(y_{1}>0\right)$ if an accident occurs. An insurance firm offers insurance contracts specifying that if a premium $x(x>0)$ is paid, the insured receives nothing if no accident occurs and receives $x+s(x)(s(x)>0)$ if an accident occurs. Thus, $s(x)$ is the compensation, above the premium, paid in case of an accident.

Individuals choose their optimum insurance contracts by maximizing expected utility $U$. We assume that all individuals have the same strictly concave von Neumann-Morgenstern utility function $u(\cdot)$, but differ with respect to the probability with which they suffer accidents. We call an individual with an accident probability of $p(1>p>0)$, a $p$-individual. Individuals are assumed to know their accident probabilities accurately. Thus, the p-individual maximizes his expected utility

$$
U(p, x)=(1-p) u\left(y_{0}-x\right)+p u\left(y_{1}+s(x)\right)
$$

with respect to $x$.

We assume that the utility function $u(\cdot)$ is twice differentiable and $u^{\prime}>0$, $u^{\prime \prime}<0$ for all $x \geq 0$. Further, in order to ensure that individuals choose to have a positive consumption in both states, we assume that $u^{\prime}(0)=\infty$. Suppose, finally, that the insurance policy $s(\cdot)$ is also twice differentiable, with $s^{\prime}>0$ for all $x \geq 0$.

The first-order condition for a maximum of (1) is then

$$
\begin{array}{r}
-(1-p) u^{\prime}\left(y_{0}-x\right)+p u^{\prime}\left(y_{1}+s(x)\right) s^{\prime}(x) \leq 0 \\
{\left[-1(1-p) u^{\prime}\left(y_{0}-x\right)+p u^{\prime}\left(y_{1}+s(x)\right) s^{\prime}(x)\right]=0}
\end{array}
$$

The second-order condition for a unique global maximum is assumed to hold

$$
(1-p) u^{\prime \prime}\left(y_{0}-x\right)+p u^{\prime \prime}\left(y_{1}+s(x)\right) s^{\prime}(x)^{2}+p u^{\prime}\left(y_{1}+s(x)\right) s^{\prime \prime}(x)<0
$$

for all $1 \geq p \geq 0$ and $y_{0}>x \geq 0$. From (2) and (3) it is seen that the optimum $x$ is increasing with $p$. Hence, given our assumptions, there exists a number $\underline{p}$, $1>\underline{p}>0$, defined by the condition

$$
-(1-\underline{p}) u^{\prime}\left(y_{0}\right)+\underline{p} u^{\prime}\left(y_{1}\right) s^{\prime}(0)=0
$$

such that the optimum of the $p$-individual $x(p)$ is given by

$$
x(p)= \begin{cases}0 & p<\underline{p} \\ \hat{x}(p) & p \geq \underline{p}\end{cases}
$$


where $\hat{x}(p)$ is determined by the condition

$$
-(1-p) u^{\prime}\left(y_{0}-\hat{x}\right)+p u^{\prime}\left(y_{1}+s(\hat{x})\right) s^{\prime}(\hat{x})
$$

By (3), (4) and (6), $\hat{x}(\underline{p})=0$ and $d \hat{x} / d p>0$ for $p>\underline{p}$; that is, the size of the optimum policy continuously increases with the probability of accident.

The basic assumption concerning the imperfect information available to the insurance firm is that it cannot distiguish among individuals according to their accident probabilities, except by their market behavior. Any policy offered is therefore open to all individuals in the market without discrimination.

For a given policy $s(\cdot)$, expected profits of the form from a $p$-individual $R[p, s(\cdot)]$, are

$$
R[p, s(\cdot)]=(1-p) x(p)-p s(x(p))
$$

where $x(p)$ is given by $(5)$.

Let there be $f(p)(f \geq 0) p$-individuals in the market. Normalizing the size of the potential customer population to unity, we may regard $f(p)$ as a density function. Total expected profits of the form, $R[s(\cdot)]$, are then given by

$$
R[s(\cdot)]=\int_{0}^{1} R[p, s(\cdot)] f(p) d p
$$

The insurance firm is assumed to have information on individuals' behavior $x(p)$ and on the density function $f(p)$, and its objective is to find the policy $s(\cdot)$ that maximizes $(7)$.

Methods of the calculus of variations can be applied to solve this problem. The standard theory, however, has to be extended in order to take into account the constraint imposed by (2). The structure of this problem is similar to the problem of the choice of an optimum income tax function [see Mirrlees (1971) and Sheshinski (1976)]. As in that problem, the first-order conditions for the appropriate maximization do not provide much insight into the method or the properties of the optimum function. We shall therefore proceed to consider special cases for which an explicit solution can be found. The first case restricts the policy chosen by the firm to be linear with a ceiling on size. The other cases allow nonlinear policies but restrict the form of the utility function.

\section{The optimum linear policy with a ceiling}

Suppose that the insurance firm offers a policy which is in fixed proportion $\beta$ $(\beta>0)$ to the premium paid, provided the size of the premium does not exceed a certain level, say, $\bar{x}^{2}$ Thus,

$$
s(x)= \begin{cases}\beta x, & x \leq \bar{x} \\ \beta \bar{x}, & x>\bar{x}\end{cases}
$$

\footnotetext{
${ }^{2}$ This violates our assumption that $s(x)$ is differentiable at $x$, but the required modification is incorporated in equation (4).
} 
If the quantity constaint $\bar{x}$ is effective, then there exist numbers $\bar{p}$ and $\underline{p}$, $1>\bar{p}>\underline{p}>0$, defined by

$$
\begin{gathered}
-(1-\bar{p}) u^{\prime}\left(y_{0}-\bar{x}\right)+\bar{p} u^{\prime}\left(y_{1}+\beta \bar{x}\right) \beta=0 \\
-(1-\underline{p}) u^{\prime}\left(y_{0}\right)+\underline{p} u^{\prime}\left(y_{1}\right) \beta=0
\end{gathered}
$$

The optimum of the $p$-individual is now given by

$$
x(p)= \begin{cases}0 & p \leq \underline{p} \\ \hat{x}(p) & \bar{p}>p \geq \underline{p} \\ \bar{x} & p \geq \bar{p}\end{cases}
$$

where $\hat{x}(p)$ is determined by (6).

$$
-(1-p) y^{\prime}\left(y_{0}-\hat{x}\right)+p u^{\prime}\left(y_{1}+\beta \hat{x}\right) \beta=0
$$

By (10) and (13), $\hat{x}(\bar{p})=\bar{x}$. Total expected profits $R(\beta, \bar{x})$ are given by

$$
R(\beta, \bar{x})=\int_{p}^{\bar{p}}[(1-p) \hat{x}(p)-p \beta \hat{x}(p)] f(p) d p+\int_{p}^{1}[(1-p) \bar{x}-p \beta m b x] f(p) d p
$$

Using (10), (11) and (13), the first-order conditions for an interior maximum of (14) w.r.t $\beta$ and $\bar{x}$ are, respectively,

$$
\begin{gathered}
\frac{\partial R}{\partial \beta}=\int_{\underline{p}}^{\bar{p}}(1-p-p \beta) \frac{\partial \hat{x}}{\partial \beta} f(p) d p-\int_{\underline{p}}^{\bar{p}} p \hat{x}(p) f(p) d p-\bar{x} \int_{\bar{p}}^{1} p f(p) d p=0 \\
\frac{\partial R}{\partial \bar{x}}=\int_{\bar{p}}^{1}(1-p) f(p) d p-\beta \int_{\bar{p}}^{1} p f(p) d p=0
\end{gathered}
$$

Differentiating (13), we have

$$
\frac{\partial \hat{x}}{\partial \beta}=\frac{-p\left[u^{\prime}\left(y_{1}+\beta \hat{x}\right)+u^{\prime \prime}\left(y_{1}+\beta \hat{x}\right) \beta \hat{x}\right]}{(1-p) u^{\prime \prime}\left(y_{0}-\hat{x}\right)+p u^{\prime \prime}\left(u_{1}+\beta \hat{x}\right) \beta^{2}}
$$

Substituting (17) into (15), using (13), we obtain

$$
\begin{aligned}
\frac{\partial R}{\partial \beta}= & \int_{\underline{p}}^{\bar{p}}\left[\frac{u^{\prime}\left(u_{0}-\hat{x}\right)-u^{\prime \prime}\left(y_{0}-\hat{x}\right) \hat{x}-\left(u^{\prime}\left(y_{1}+\beta \hat{x}\right)+u^{\prime \prime}\left(y_{1}+\beta \hat{x}\right) \beta \hat{x}\right)}{(1-p) u^{\prime \prime}\left(y_{0}-\hat{x}\right)+p u^{\prime \prime}\left(y_{1}+\beta \hat{x}\right) \beta^{2}}\right] \\
& \times p(1-p) f(p) d p-\bar{x} \int_{\bar{p}}^{1} p f(p) d p=0
\end{aligned}
$$


Equations (10), (11), (16), and (18) determine the profit-maximizing values of $\bar{p}, p, \bar{x}$, and $\beta$. Notice that for small $\beta$, the second term in (18) is small, and the sign of (18) is then the same as the sign of $u^{\prime}\left(y_{1}\right)-u^{\prime}\left(y_{0}\right)$, which is positive, assuming that $y_{0}>y_{1}$.

Obviously, depending on $y_{0}, y_{1}$ and the function $f(p)$, conditions on $u(\cdot)$ may have to be imposed to ensure the existence of an interior optimum and the second-order conditions. We shall explore these conditions for a special case of the above model.

Let $u(z)=\log z$. For this case, by (13), $\hat{x}=p y_{0}-\left[(1-p) y_{1} / \beta\right]$. By (10) and (11), we have

$$
\underline{p}=y_{1} /\left(\beta y_{0}+y_{1}\right), \quad \bar{p}=\left(y_{1}+\beta \bar{x}\right) /\left(\beta y_{0}+y_{1}\right)
$$

and the first order condition (18) becomes

$$
\frac{\partial R}{\partial \beta}=\int_{\underline{p}}^{\bar{p}}\left[\left(\frac{1-p}{\beta}\right)^{2} y_{1}-p^{2} y_{0}\right] f(p) d p-\bar{x} \int_{\bar{p}}^{1} p f(p) d p=0
$$

Equations (16), (19), and (20) determine the optimum values of $p, \bar{p}, \bar{x}$, and $\beta$. From (20), the optimum $\beta$ satisfies

$$
\beta=\left[\frac{y_{1} \int_{\underline{p}}^{\bar{p}}(1-p)^{2} f(p) d p}{y_{0} \int_{\underline{p}}^{p} p^{2} f(p) d p+\bar{x} \int_{\bar{p}}^{1} p f(p) d p}\right]^{1 / 2}
$$

In order to have $\beta>0$, it is necessary (as assumed) that $y_{1}>0$. It can be shown that there are values of $y_{0}$ and $y_{1}$ which yield an interior solution and that satisfy the second-order conditions.

\section{Comparison with the socially optimal policy}

We want to compare the insurance policy chosen by the monopoly with a policy which is socially optimal under the same informational structure. Thus, the social planner, as the firm, cannot identify individuals by their accident probability, but knows the aggregate distribution $f(p)$ and the relation $\hat{x}(p)$. We adopt the utilitarian objective, where social welfare $W$ is given by the sum of individuals' expected utilities:

$$
\begin{aligned}
W= & \int_{0}^{1} U(p) f(p) d p=\int_{0}^{\underline{p}}\left[(1-p) u\left(y_{0}\right)+p u\left(y_{1}\right)\right] f(p) d p \\
& +\int_{\underline{p}}^{\bar{p}}\left[(1-p) u\left(y_{0}-\hat{x}\right)+p u\left(y_{1}+\beta \hat{x}\right)\right] f(p) d p \\
& +\int_{\bar{p}}^{1}\left[(1-p) u\left(y_{0}-\bar{x}\right)+p u\left(y_{1}+\beta \bar{x}\right)\right] f(p) d p
\end{aligned}
$$


Consider the problem of maximizing (22) w.r.t $\beta$ and $\bar{x}$, subject to a budget constraint

$$
\int_{\underline{p}}^{\bar{p}}[(1-p) \hat{x}(p)-p \beta \hat{x}(p)] f(p)+\int_{\bar{p}}^{1}[(1-p) \bar{x}-p \beta \bar{x}] f(p) d p-\bar{R}=0
$$

for a given $\bar{R}>0$. To make the socially optimal policy comparable with the monopoly's policy, $\bar{R}$ should be equal to the maximum value of (14).

Using (10)-(13), the first-order conditions for an interior maximum can be written

$$
\begin{gathered}
\frac{\partial W}{\partial \beta}=\mu \frac{\partial R(\beta, \bar{x})}{\partial \beta}+\int_{\underline{p}}^{\bar{p}} u^{\prime}\left(y_{1}+\beta \hat{x}\right) \hat{x} p f(p) d p+u^{\prime}\left(y_{1}+\beta \bar{x}\right) \bar{x} \int_{\bar{p}}^{1} p f(p) d p=0 \\
\frac{\partial W}{\partial \bar{x}}=\mu \frac{\partial R(\beta, \bar{x})}{\partial \beta}+\int_{\bar{p}}^{1}\left[-(1-p) u^{\prime}\left(y_{0}-\bar{x}\right)+p u^{\prime}\left(y_{1}+\beta \bar{x}\right) \beta\right] f(p) d p=0
\end{gathered}
$$

where $\mu>0$ is a constant (Lagrange multiplier).

In order to compare the socially optimum values of $\beta$ and $\bar{x}$ determined by the system (10), (11), (13), (24), and (25) with the values chosen by the monopoly, we need to determine the sign of $\partial^{2} R / \partial \beta \partial \bar{x}$. By (16),

$$
\frac{\partial^{2} R}{\partial \beta \partial \bar{x}}=(\beta \bar{p}+\bar{p}-1) f(\bar{p}) \frac{\partial \bar{p}}{\partial \beta}-\int_{\bar{p}}^{!} p f(p) d p
$$

Since $\partial^{2} R / \partial \bar{x}^{2}=(\beta \bar{p}+\bar{p}-1) f(\bar{p}) \partial \bar{p} / \partial \bar{x}<0$ and, by $(10), \partial \bar{p} / \partial \bar{x}>0$, it follows that $\beta \bar{p}+\bar{p}-1<0$. Now, differentiating (10), we obtain

$$
\frac{\partial \bar{p}}{\partial \beta}=\frac{-\bar{p}\left[u^{\prime}\left(y_{1}+\beta \bar{x}\right)+u^{\prime \prime}\left(y_{1}+\beta \bar{x} \beta \bar{x}\right)\right]}{u^{\prime}\left(y_{0}-\bar{x}\right)+u^{\prime}\left(y_{1}+\beta \bar{x}\right) \beta}
$$

Hence, the sign of (26) is negative provided $\partial \bar{p} / \partial \beta \geq 0$. The latter holds, by (27), when $u^{\prime \prime}(z) z / u(z) \leq 1$.

Assume, therefore, that $\partial^{2} R / \partial \beta \partial \bar{x}<0$. Since the last two terms in (24) and the last term in (25) are positive, the position of the curves $\partial W / \partial \beta=0$ and $\partial W / \partial \bar{x}=0$ relative to the curves $\partial R / \partial \beta=0$ and $\partial R / \partial \bar{x}=0$, is as indicated in Figure 1. (The way in which these curves intersect is determined by secondorder conditions.) The monopoly optimum is indicated by $M$ and the social optimum by $P$.

It is seen that the social optimum provides a higher marginal return $\beta$, or a higher ceiling $\bar{x}$, or both, compared with the policy chosen by the monopoly. Clearly, the social optimum cannot have a lower marginal return and a lower ceiling than the monopoly's policy. 


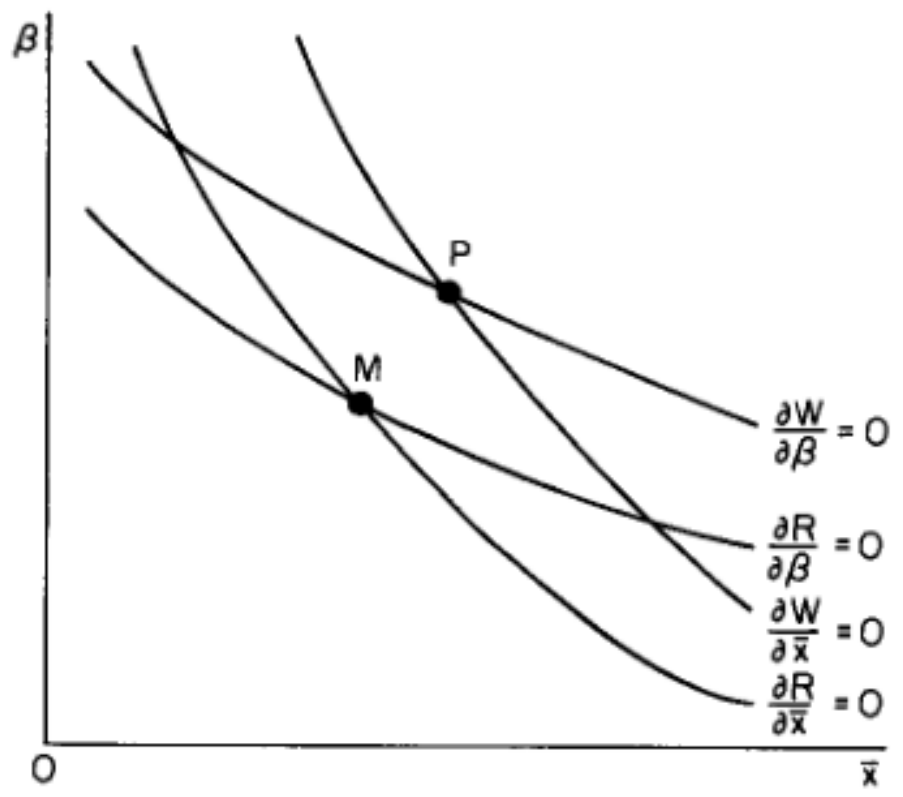

Figure 1:

\section{A nonlinear example}

We shall now consider a case in which, for a particular class of utility and density functions, an explicit nonlinear optimum policy can be derived.

Let $u(y)=y^{1 / 2}$ and define $Y$ and $S$ to be

$$
Y=\left(y_{0}-x\right)^{1 / 2}, \quad S=\left(y_{1}+s(x)\right)^{1 / 2}
$$

Expected utility is then

$$
U=(1-p) Y+p S
$$

in the $Y S$ plane indifference curves are straight lines with slope $\pi \equiv(1-p) / p$. Since $Y$ and $S$ are strictly monotone functions of $x$, one can write $S$ uniquely in terms of $Y, S=S(Y)$. For the individual to have a unique optimum, the function $S(Y)$ has to be strictly concave. We shall have to check later whether the optimum solution satisfies this condition.

The $p$-individual's maximization condition is

$$
S^{\prime}(Y)=-\pi
$$

which, together with the constraint $S=S(y)$, solves for the optimum pair $(S(\pi), Y(\pi))$. From $(30)$,

$$
d S / d \pi=-\pi^{d Y} / d \pi
$$


By (7), (8) and (28), expected profits are given by

$$
R[s(\cdot)]=\int_{0}^{\infty}\left[y_{0} \pi+y_{1}-Y^{2}(\pi) \pi-S^{2}(\pi)\right] g(\pi) d \pi
$$

where $g(\pi) d \pi=-p f(p) d p$. Since $y_{0} \pi+y_{1}$ is not controlled by the firm, its objective can be restated as

$$
\min _{S(Y)} \int_{0}^{\infty}\left[Y^{2}(\pi) \pi+S^{2}(\pi)\right] g(\pi) d \pi
$$

subject to (11). Forming the Lagrangean function, the Euler equations with respect to $S(\pi)$ and $Y(\pi)$ are

$$
\begin{gathered}
\frac{d}{d \pi}[\lambda(\pi) g(\pi)]=2 S(\pi) g(\pi) \\
\frac{d}{d \pi}[\lambda(\pi) \pi g(\pi)]=2 Y(\pi) \pi g(\pi)
\end{gathered}
$$

where $\lambda(\pi)$ is the "shadow-price" function. Combining (34) and (35), we may write these as

$$
(d / d \pi)[(Y(\pi)-S(\pi)) \pi g(\pi)]=S(\pi) g(\pi)
$$

Equations (36) and (31) form two first-order differential equations which may be solved for $Y(\pi)$ and $S(\pi)$, ince $\mathrm{mg}(\pi)$ is specified.

As an example, consider the density function $g(\pi)=1 / \pi$. By definition, this implies that $f(p)=1 /(1-p) p^{3}$, which is a U-shaped density with concentration of individuals at the two extremes. Condition (36) become

$$
(d Y / d \pi)-(d S / d \pi)=S / \pi
$$

Substituting (31) in (37), we have

$$
1 / S d S / d \pi=-1 /(1+\pi)
$$

with solution

$$
S(\pi)=a /(1+\pi)
$$

for some constant $a>0$. From (19) and (11),

$$
d Y / d \pi=a / \pi(1+\pi)^{2}
$$

with solution

$$
Y(\pi)=b+\frac{a}{1+\pi}+a \log \left(\frac{\pi}{1+\pi}\right)
$$

for some $b>0$. When $\pi \rightarrow \infty(p \rightarrow 0), Y(\pi) \rightarrow b$. Since $p \rightarrow 0$ implies $x \rightarrow 0$ and hence $Y(\pi) \rightarrow y_{0}^{1 / 2}$, it follows that $b=y_{0}^{1 / 2}$. 
To make sure that $Y>0\left(y_{0}>x\right)$, we assume that there is a positive lower bound on $\pi$, denoted $\pi_{0}$, which satisfies $Y\left(\pi_{0}\right)>0$.

The final step is to minimize (33) with respect to $a$, but we shall delete this condition.

Notice also that the concavity of $S(Y)$, required for the uniqueness of the individual maximization solution (30), is satisfied. By (39) and $(40), d^{2} S / d Y^{2}=$ $-a / \pi(1-\pi)^{2}<0$.

Equations (39), (31), and (28) determine implicitly the optimum policy $s(x)$. Differentiating, using (28), (38), and (40), we obtain

$$
\frac{d s}{d x}=\frac{d s / d \pi}{d x / d \pi}=-\frac{S(\pi) d S / d \pi}{Y(\pi) d Y / d \pi}=\frac{S(\pi) \pi}{Y(\pi)}>0
$$

Also, using (39)-(42), we have

$$
\begin{aligned}
\frac{d}{d \pi}\left(\frac{d s}{d x}\right) & =\frac{S(\pi)}{Y^{2}(\pi)(1+\pi)}\left[Y(\pi)-\frac{a}{1+\pi}\right]=\frac{S(\pi)}{Y^{2}(\pi)(1+\pi)}[Y(\pi)-S(\pi)] \\
& =\frac{S(\pi)}{Y^{2}(\pi)(1+\pi)}\left[\left(y_{0}-x\right)^{1 / 2}-\left(y_{1}+s(x)\right)^{1 / 2}\right] \\
& =\frac{S(\pi)}{Y^{2}(\pi)(1+\pi)}\left[b+a \log \left(\frac{\pi}{1+\pi}\right)\right]
\end{aligned}
$$

Since $d x / d p<0$, we have from (43) that $d^{2} s / d x^{2}$ has the opposite sign of $Y-S=$ $\left(y_{0}-x\right)^{1 / 2}-\left(y_{1}+s(x)\right)^{1 / 2}$. Assume that $y_{0}>y_{1}$ (income in case of accident lower than with no accident). If individuals choose to have in case of accident an income inclusive of insurance premium that does not exceed income net of insurance payment in case of no accident, then $Y-S \geq 0$. It would then follow that $d^{2} s / d x^{2}<0$. However, one can find parameter values in the above example that will induce individuals with high accident probability (small $\pi$ ) to choose to insure themselves for a higher income in case of an accident than their income

in the absence of one. In such a case, $d^{2} s / d x^{2}$ is positive at large $x$ (and negative at smaller $x$ ).

\section{References}

[1] Arrow, K. J. (1973). Higher Education as a Filter, Journal of Public Economics 2, 193-216.

[2] Mirrlees, J. A. (1971). An Exploration in the Theory of Optimum Income Taxation, Review of Economic Studies 38, 175-208.

[3] Rothschild, M., and Stiglitz, J. E. (1976). Equilibrium in Competitive Insurance Markets: An Essay on the Economics of Imperfect Information, Quarterly Journal of Economics 90, 629-650.

[4] Salop, J., and Salop, S. (1976). Self-Selection and Turnover in the Labor Market, Quarterly Journal of Economics 90, 619-628. 
[5] Sheshinski, E. (1976). An Example of Income Tax Schedules Which Are Optimal for the Maxi-Min Criterion, International Economic Review (forthcoming).

[6] Spence, M. A. (1974). Market Signalling. Cambridge, Massachusetts: Harvard Univ. Press. 